\title{
Different brain networks underlying intelligence in autism spectrum disorders
}

\author{
Emmanuel Peng Kiat Pua ${ }^{1,2}$ (1) | Charles B. Malpas ${ }^{1,2,3}$ | Stephen C. Bowden ${ }^{1,4}$ | \\ Marc L. Seal ${ }^{2,5}$ \\ ${ }^{1}$ Melbourne School of Psychological Sciences, University of Melbourne, Parkville, Victoria 3010, Australia \\ ${ }^{2}$ The Royal Children's Hospital, Developmental Imaging, Murdoch Children's Research Institute, The Royal Children's Hospital, Victoria 3052, Australia \\ ${ }^{3}$ Clinical Outcomes Research Unit, Department of Medicine, Royal Melbourne Hospital, The University of Melbourne, Parkville, Victoria 3010, Australia \\ ${ }^{4}$ St. Vincent's Hospital, 41 Victoria Parade, Fitzroy, Victoria 3065, Australia \\ ${ }^{5}$ Department of Paediatrics, University of Melbourne, Parkville, Victoria 3010, Australia
}

\section{Correspondence}

Emmanuel Pua, Developmental Imaging, Murdoch Children's Research Institute, The Royal Children's Hospital, Victoria 3052, Australia.

Email: emmanuel.pua@mcri.edu.au

\section{Funding information}

National Institute of Mental Health, Grant/ Award Number: NIMH 5R21MH107045; Murdoch Children's Research Institute, the Royal Children's Hospital, Department of Paediatrics, The University of Melbourne and the Victorian Government's Operational Infrastructure Support Program; The Royal Children's Hospital Foundation, Grant/Award Number: $\mathrm{RCH} 1000$

\begin{abstract}
There has been sustained clinical and cognitive neuroscience research interest in how network correlates of brain-behavior relationships might be altered in Autism Spectrum Disorders (ASD) and other neurodevelopmental disorders. As previous work has mostly focused on adults, the nature of whole-brain connectivity networks underlying intelligence in pediatric cohorts with abnormal neurodevelopment requires further investigation. We used network-based statistics (NBS) to examine the association between resting-state functional Magnetic Resonance Imaging (fMRI) connectivity and fluid intelligence ability in male children $(n=50)$ with Autism Spectrum Disorders (ASD; $M=$ $10.45, S D=1.58$ years and in controls $(M=10.38, S D=0.96$ years) matched on fluid intelligence performance, age and sex. Repeat analyses were performed in independent sites for validation and replication. Despite being equivalent on fluid intelligence ability to strictly matched neurotypical controls, boys with ASD displayed a subnetwork of significantly increased associations between functional connectivity and fluid intelligence. Between-group differences remained significant at higher edge thresholding, and results were validated in independent-site replication analyses in an equivalent age and sex-matched cohort with ASD. Regions consistently implicated in atypical connectivity correlates of fluid intelligence in ASD were the angular gyrus, posterior middle temporal gyrus, occipital and temporo-occipital regions. Development of fluid intelligence neural correlates in young ASD males is aberrant, with an increased strength in intrinsic connectivity association during childhood. Alterations in whole-brain network correlates of fluid intelligence in ASD may be a compensatory mechanism that allows equal task performance to neurotypical peers.
\end{abstract}

\section{1 | INTRODUCTION}

Fluid intelligence refers to the ability to solve novel problems, and is typically estimated from composite scores of nonverbal or abstract tests. Under the Catell-Horn-Carroll (CHC) model, fluid intelligence (Gf) is a broad ability subsumed under an overall general intelligence factor (Reynolds \& Keith, 2017; Schneider \& McGrew, 2012). The autism spectrum disorders (ASD) are a group of heterogeneous neurodevelopmental conditions associated with deficits in social communication, social interaction, and restricted and repetitive behaviours. Uneven subtest scores within IQ measures have been observed across high- and low functioning ASD populations, as well as in both children and adults with ASD (Happé, 1994). In particular, nonverbal fluid intelligence performance in ASD groups has been suggested to be elevated relative to crystallized intelligence scores on verbal tasks. However, a neurobiological account of brain-behavior associations in intelligence ability in ASD is lacking, and 
the link between altered neurodevelopment in ASD and cognitive performance remains poorly understood (Ehlers, et al., 1997; Happé, 1994; Hayashi, et al., 2008).

Previous investigations on the neural correlates of ASD fluid intelligence ability have primarily relied on task-based paradigms in functional Magnetic Resonance Imaging (fMRI), based on blood oxygen level dependent (BOLD) response as an estimate of local brain regions recruited when performing a particular task. Adults with ASD demonstrate an abnormal reliance on enhanced visuospatial processes in extrastriate and parietal regions when engaging in fluid tasks (Koshino, et al., 2005; Mottron, et al., 2013). Increase in fluid task complexity modulated stronger activity in occipital and temporal regions, coupled with higher connectivity between major lobar regions (Simard, et al., 2015; Soulières, et al., 2009). Fluid intelligence performance in neurotypical individuals is known to involve broad recruitment across frontal, parietal, temporal and occipital cortices, as well as subcortical striatal and thalamic regions (Burgaleta, et al., 2014; Geake \& Hansen, 2010; Gong, et al., 2005; Kroger, et al., 2002; Perfetti, et al., 2009; Prabhakaran, et al., 1997). In contrast, connectivity to prefrontal cortical areas observed in controls during fluid tasks were either altered or absent in adults with ASD, suggesting impairments in functional segregation and integration in neural mechanisms underlying ASD fluid intelligence ability primarily characterized by increased occipito-parietal and temporal activity (Sahyoun, et al., 2010; Yamada, et al., 2012).

Previous studies focused on localization may be associated with a bias towards identification of task-positive regions, and there is a need for network-based whole-brain investigations (Basten, et al., 2015; Langeslag, et al., 2013). One approach to elucidate the neural correlates of fluid intelligence is the use of resting-state or intrinsic functional connectivity, defined as the temporal synchronicity of BOLD time-series between spatially distinct brain regions when no task is being performed (Lowe, et al., 2016). Intrinsic functional connectivity offers powerful methods to characterize the functional architecture of the brain. The measure corresponds to individual differences during taskdependent active states, and has also been shown to predict fluid intelligence ability (Smith, et al., 2009; Tavor, et al., 2016). However, most investigations on brain networks underlying cognition tend to be limited to general intelligence or neurotypical adult populations (Haász, et al., 2013; Malpas, et al., 2016; Penke, et al., 2012). The nature of whole-brain fluid intelligence connectivity networks in neurodevelopmental disorders is thus not well understood in pediatric populations. Specifically, it is not clear how the neurocognitive architecture of fluid intelligence is altered in ASD during early stages of cognitive development (Gray, et al., 2003; Kosslyn, et al., 2002).

To address this gap, we investigated whether whole-brain intrinsic functional connectivity networks associated with fluid intelligence were altered in children with ASD. Comparisons for all analyses were made to typically developing controls matched on age, sex, and fluid intelligence ability. Importantly, strict matching criteria was implemented to ensure that observed differences were not likely to be explained by common confounds faced in ASD research (Pua, et al., 2017).

\section{2 | MATERIALS AND METHODS}

\section{1 | Participants}

Data was obtained from the Kennedy Krieger Institute (KKI, ABIDE-II) sample from the Autism Brain Imaging Database Exchange (ABIDE; Di Martino, et al., 2014). ABIDE sites were only selected if comprehensive fluid intelligence measures were available, and age ranges and sample sizes were suitable for analyses (See Supporting Information Figure S1). Following this criteria, the KKI site was included for the main analysis. Participants in the KKI sample were recruited as part of a study run by the Center for Neurodevelopment and Imaging Research (CNIR) at the $\mathrm{KKI}$. All eligible participants received an MRI scan and cognitive assessment with the Wechsler Intelligence Scale for Children (Fourth Edition, WISC-IV; Fifth Edition, WISC-V). Handedness was assessed using the Edinburgh Handedness Inventory. Inclusion criteria were an age range of 8 years and 0 months to 12 years, 11 months and 30 days, and WISC-IV or WISC-V Full Scale Intelligence Quotient $>80$. Diagnosis of ASD was determined using the Autism Diagnostic Interview-Revised (ADI-R), Autism Diagnostic Observation Schedule-Generic (ADOS-G) module 3 or the ADOS-2 module 3 . ASD classification criteria was based on the ADOS-G and/or ADI-R and clinical assessment by an expert pediatric neurologist with extensive experience in autism diagnosis. ASD participants were excluded if they had an identifiable cause of autism. For the control group, participants with a history of developmental or psychiatric disorders or with a first-degree relative with ASD were excluded. Full protocol details for sampling, image acquisition and phenotyping for all ABIDE sites are available elsewhere ${ }^{1}$.

For the present study, inclusion criteria applied to the KKI sample were male participants satisfying DSM-IV-TR ${ }^{2}$ Pervasive Developmental Disorder criteria (Autistic Disorder, Asperger's or Not Otherwise Specified), assessed with the WISC-IV, and with MRI data acquired under the same scanning protocol. Continuous variables in the phenotype data were demeaned. Nonparametric propensity matching was performed using the Matchlt package (Ho, et al., 2011) in the $R$ environment (Team, 2014). Male participants with ASD were matched with TD controls on the following variables: sex, age in years [ASD: $M=10.45$ (SD = 1.58); TD: $M=10.38$ (SD = 0.96)], and PRI score (Perceptual Reasoning Index, or fluid intelligence score estimate) from the WISC-IV [ASD: 108.65 (13.57); TD: 108.83 (14.14)]. The matching procedure resulted in a final sample of 50 male participants (ASD: $n=26$; TD: $\mathrm{n}=24$ )

To evaluate if findings could be replicated, we repeated the same image processing and analysis on independent samples from other ABIDE sites. The Georgetown University (GU) site was selected based on similarity to the $\mathrm{KKI}$ sample in the main analyses in cohort age range (8 to 13 years), availability of fluid intelligence measures, and adequate

${ }_{1}^{1}$ Public access ABIDE protocol: http://fcon_1000.projects.nitrc.org/indi/ abide/.

${ }^{2}$ DSM-IV-TR: Diagnostic and statistical manual of mental disorders, text revision. American Psychiatric Association, \& American Psychiatric Association. (2000). DSM-IV-TR: Diagnostic and statistical manual of mental disorders, text revision. Washington, DC: American Psychiatric Association, 75. 
TABLE 1 Descriptive statistics of samples by site

\begin{tabular}{|c|c|c|c|c|c|}
\hline Site & Sample size & Age in years $[M(S D)]$ & Age range in years & PRI [M(SD)] & Test type \\
\hline \multicolumn{6}{|l|}{ KKI } \\
\hline ASD & 26 & 10.45 (1.58) & 8 to 13 & $108.65(13.57)$ & WISC-IV \\
\hline TD & 24 & $10.38(0.96)$ & 8.9 to 12.8 & $108.83(14.14)$ & WISC-IV \\
\hline \multicolumn{6}{|l|}{ GU } \\
\hline ASD & 25 & $11.04(1.44)$ & 8.28 to 13.08 & $109.68(14.06)$ & WASI \\
\hline TD & 25 & $10.6(1.46)$ & 8.06 to 12.7 & $118.52(13.45)$ & WASI \\
\hline ASD & 15 & 20.32 (1.48) & $18.41-22.88$ & $102.67(13.98)$ & WASI \\
\hline TD & 15 & $18.46(2.45)$ & $15-23.95$ & 111 (10.39) & WASI \\
\hline
\end{tabular}

Note. $\mathrm{ASD}=$ Autism Spectrum Disorders; GU = Georgetown University; KKI = Kennedy Krieger Institute; $\mathrm{M}=$ mean; PRI = Perceptual Reasoning Index, or fluid intelligence score estimate; $S D=$ standard deviation, TD = typically developing; USM = The University of Utah School of Medicine;

WASI = Wechsler Abbreviated Scale of Intelligence; WISC-IV = Wechsler Intelligence Scale for Children - Fourth Edition.

sample size. Because imaging acquisition protocols and parameters, and behavioral test measures differed between sites, GU was used as a validation sample rather than collapsed with the initial analyses. To investigate if similar results could be observed in an older age cohort (age range: 15 to 24 years), the same analysis was repeated in The University of Utah School of Medicine (USM) sample. Table 1 provides descriptive statistics for all samples used for present analyses.

\section{2 | Image acquisition}

MRI data was acquired on a 3 Tesla Phillips scanner (Achieva; Philips Healthcare, Best, The Netherlands). T1-weighted images were obtained through a 200 slice three-dimensional acquisition (Turbo Field Echo [TFE] MPRAGE; acquisition time $=8 \mathrm{~min} 8 \mathrm{sec}$; coronal slice orientation; flip angle $=8^{\circ}$; repetition time $(T R)=8 \mathrm{~ms}$; echo time $(T E)=3.7$ $\mathrm{ms}$; minimum inverse time (TI) delay $=843.25 \mathrm{~ms}$; field of view $(F O V)=256 ;$ matrix $=256 \times 200$; slice thickness $=1 \mathrm{~mm}$, in-plane resolution $=1 \mathrm{~mm} \times 1.28 \mathrm{~mm}$ ). BOLD-weighted resting-state functional MRI volumes were acquired using echo planar imaging (EPI; number of volumes $=128$; $\mathrm{TE}=30 \mathrm{~ms} ; \mathrm{TR}=2500 \mathrm{~ms}$; slices $=47$; flip angle $=7^{\circ} ; \mathrm{FOV}=256$; matrix $=84 \times 81$; slice thickness $=3 \mathrm{~mm}$, inplane resolution $=3 \mathrm{~mm} \times 3.1 \mathrm{~mm}$; transverse slice orientation). During the resting-state scan, participants were instructed to relax and focus on a crosshair while remaining as still as possible with their eyes open. For the structural scan, participants watched a movie of their choice. Images were inspected after each processing step for quality control.

\subsection{Image processing and analysis}

Functional connectivity analysis and visualizations were generated with the Functional Connectivity Toolbox v.16.b (Whitfield-Gabrieli \& Nieto-Castanon, 2012) pipeline, Matlab R2010b (The MathWorks, Inc., Natick, MA) and NeuroMArVL (http://immersive.erc.monash.edu.au/ neuromarvl/). The initial four functional volumes per session were removed to account for T1 saturation effects. Slice-timing correction and first-volume realignment (using a six rigid-body parameter spatial transformation) were applied to adjust for temporal and motion artefacts. Functional volumes were normalized to MNI-space, and smoothed with a full-width half-maximum Gaussian kernel of $8 \mathrm{~mm}$. Structural images were co-registered and segmented into grey-matter, white-matter and cerebrospinal fluid for later use in the removal of physiological noise from the functional volumes. In the first-level BOLD model, realignment transformation matrices and global signal intensities were analyzed using the Artifact Detection Tool (ART) to detect motion and signal outliers. Noise from sources such as cardiac, respiratory, and other physiological activity that were not likely to be modulated by neural activity were identified using the component-based noise correction aCompCor method (Behzadi, et al., 2007). Confounds were removed by regressing artefactual effects from the BOLD signal (within-subject covariates from realignment parameters, nonneuronal physiological noise from white-matter and cerebrospinal fluid masks). Residual BOLD time series were detrended and band-pass filtered $(0.008-0.09 \mathrm{~Hz})$ before computing connectivity measures. Regions-ofinterest (ROI) were defined using the FSL Harvard-Oxford Atlas (http://www.fmrib.ox.ac.uk/fsl/) for cortical and subcortical areas, and the Anatomical Automatic Labelling (AAL) atlas (Tzourio-Mazoyer, et al., 2002) for cerebellar regions, resulting in 132 ROls. The mean BOLD time series for all voxels in each ROI were extracted to compute pairwise correlations between all ROls with the Fisher r-to-z transformation to construct a $132 \times 132$ connectivity matrix.

Network-based statistic (NBS) was used to identify brain networks showing between-group differences in functional connectivity associated with fluid intelligence performance (Zalesky, et al., 2010). NBS is a network-specific approach that is typically used to identify groups of connections showing a significant effect of interest, while controlling for the family-wise error (FWE) rate. As a nonparametric method for connectome-wide analysis, NBS offers substantially greater statistical power than generic mass-univariate testing procedures at the edge level, if connections associated with the effect of interest form connected components (defined here as interconnected subnetworks). NBS estimates an FWE-corrected $p$-value for identified subnetworks through permutation testing. Consequently, the null hypothesis can only be rejected at the level of entire subnetworks, and inferences about specific connections will not be valid. The principle behind NBS is similar to cluster-based approaches used to perform inference on 
A
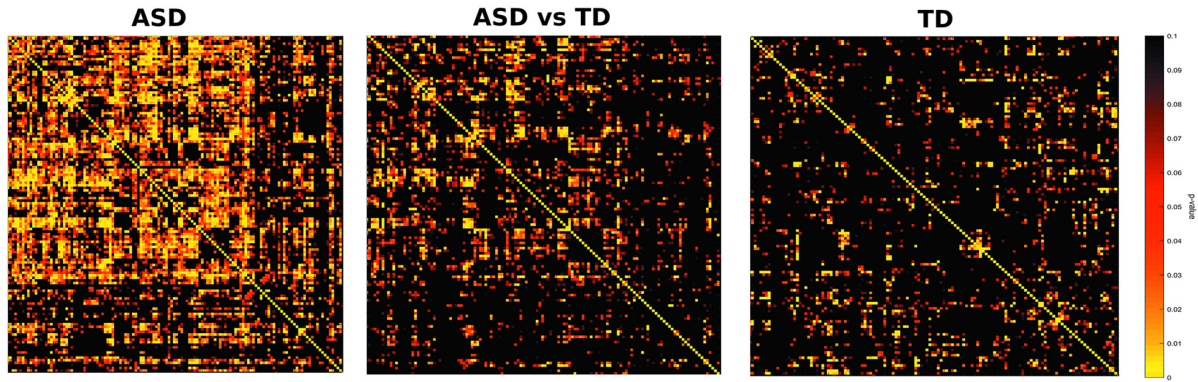

B
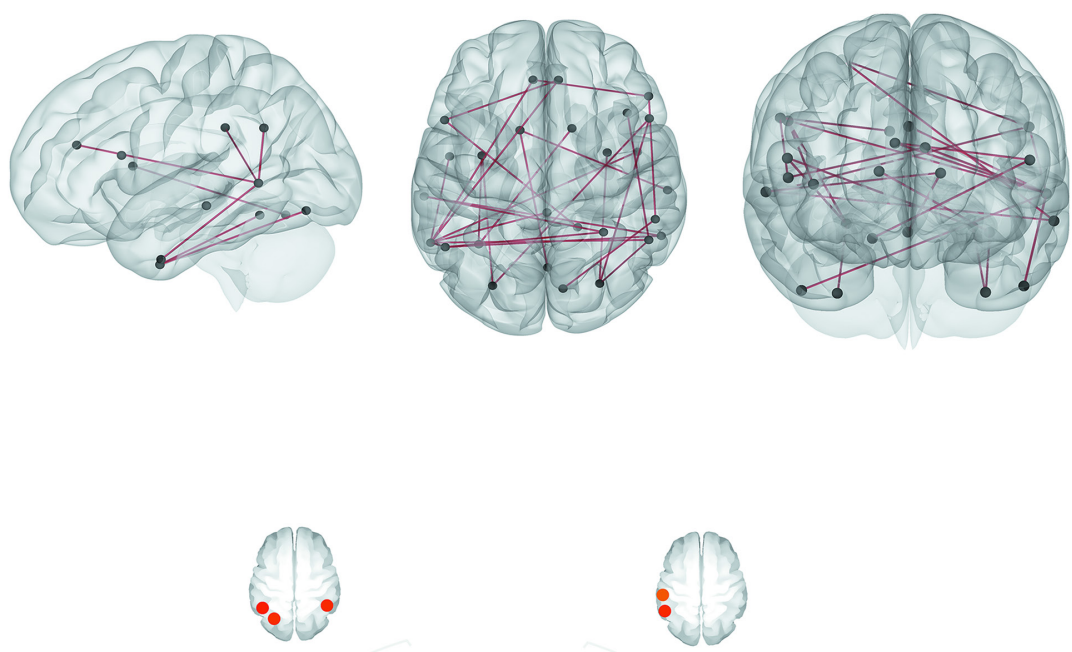

C1
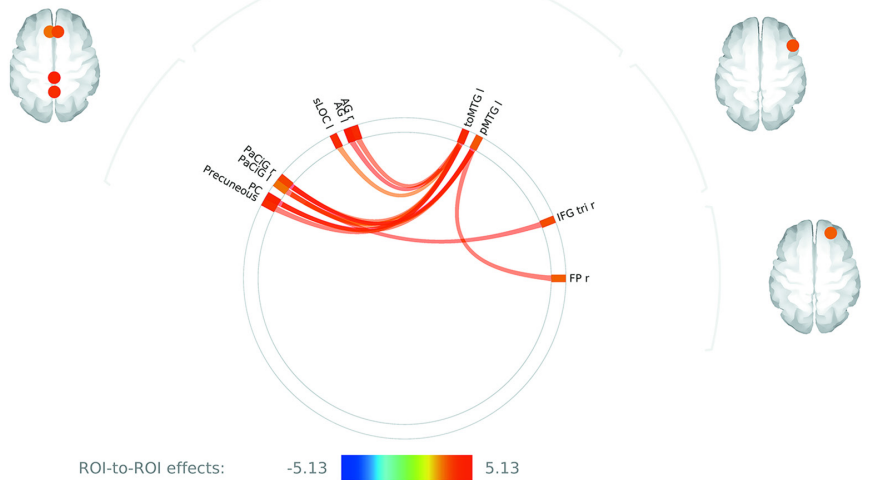

FIGURE 1 (a) $p$-value heatmaps representing second-level within-group associations of pairwise BOLD ROI-ROI edges (132 $\times 132$ matrix with fluid intelligence performance (Site: KKI). ASD: Autism Spectrum Disorders group; ASD vs TD: Group interactions between functional connectivity and fluid intelligence performance association; KKI: Kennedy Krieger Institute; TD: matched typically developing controls. (b) Visual model of subnetwork representing within-group associations in functional connectivity and fluid intelligence performance in ASD. (c) Effect of increased thresholding in network-based analysis (NBS) before network construction. In c1, only pairwise edge association with fluid intelligence of at least $t=3.5$ were retained for network construction. In $c 2$, the $t$-statistic threshold was further increased to $t=4$. AG: angular gryus; FP: frontal pole; IFG tri: inferior frontal gyrus, pars triangularis; PaCiG: paracingulate gyrus; PC: posterior cingulate gyrus; sLOC: Lateral occipital cortex, superior division; toMTG: temporo-occipital middle temporal gyrus [Color figure can be viewed at wileyonlinelibrary.com]

statistical parametric maps, and utilizes the observation that pathological effects of disease are frequently distributed across the brain but also constrained by anatomical network topology (Fornito, et al., 2016). NBS has been widely used to study altered brain connectivity in disorders such as schizophrenia (Zalesky, et al., 2011), depression (Korgaonkar, et al., 2014), addiction (Hong, et al., 2013), Parkinson's disease (Aarabi, et al., 2015), as well as connectivity correlates of cognition in the general population (e.g., Hearne, et al., 2016b).
For the present study, fluid intelligence performance scores were first regressed onto individual edges in the functional connectivity matrix. Between-group differences in functional connectivity associated with performance scores were evaluated at the second-level. Handedness and age were included as covariates for all analyses. To identify interconnected subnetworks across groups, a breadth first search (Ahuja, et al., 1993) was performed among connections surviving a t-statistic threshold (i.e., the component forming threshold) of at 


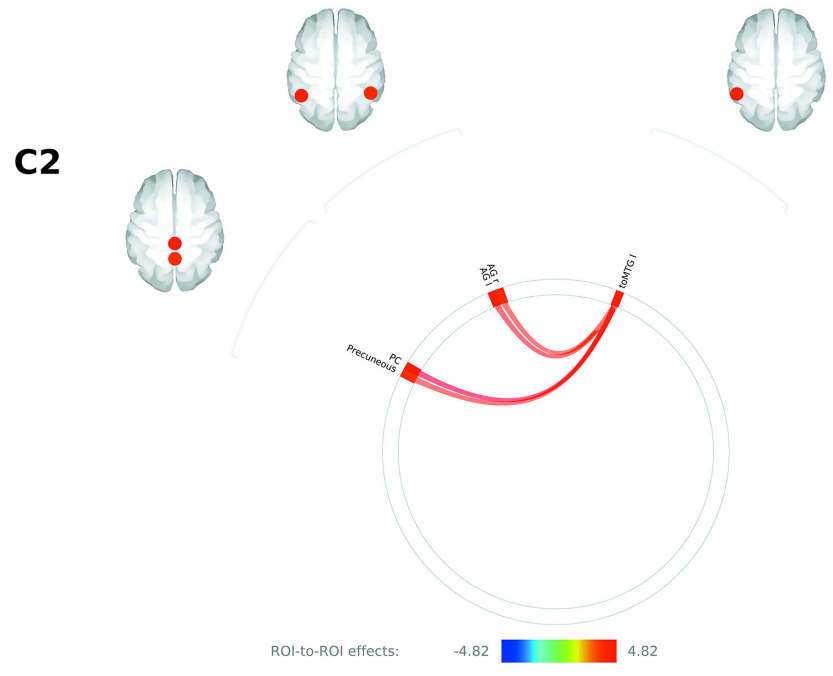

FIGURE 1 Continued

least $\mathrm{t}=3.0$, and permuted to generate a null distribution of largest network sizes. Each permutation randomly reassigns group labels and identifies the size of the largest interconnected subnetwork to yield an empirical null distribution of maximal component sizes. The estimated FWE-corrected $p$-value for an identified subnetwork of size $m$ reflects the proportion of permutations for which the largest subnetwork size is equal to or greater than $m$. The FWE rate is therefore controlled nonparametrically using a randomized null distribution of maximum component size. Subnetworks with a corrected $p$-FWE $<0.05$ value were retained.

\section{3 | RESULTS}

Between-group differences in the association of resting-state fMRI subnetwork connectivity with fluid intelligence performance was identified in males aged 8 to 13 years (network size $=24$ links, $p=$ .0373, FWE-corrected), with stronger association with fluid intelligence in ASD compared to typically developing matched controls (Figure 1). This network showing stronger correlation with fluid intelligence in ASD compared to TD involved regions in the left temporo-occipital middle temporal gyrus, left posterior middle temporal gyrus, bilateral paracingulate gryus, posterior cingulate gyrus, right frontal pole, right inferior frontal gyrus pars triangularis, bilateral angular gyrus, left lateral occipital cortex (superior division), and precuneus (Table 2). Betweengroup differences remained significant even at a more stringent component-defining threshold $(\mathrm{t}=|4|$, size $=6, p=.0425$, FWE-corrected), with nodes of the left temporo-occipital middle gyrus, bilateral angular gyrus, precuneus, and posterior cingulate gyrus that survived more conservative analyses.

Repeat analyses in samples from independent sites presented a similar pattern of findings in a cohort of comparable age (site GU; age range 8 to 13 years), showing a single fluid intelligence subnetwork with increased association in ASD compared to controls (network size $=14$ links, $t$-statistic threshold $=3.5, p=.0396$, FWE-corrected, alternate hypothesis: ASD > controls; Figure 2). Implicated regions were the bilateral occipital pole, right temoporo-occipital middle temporal gyrus, right anterior middle temporal gyrus, left posterior middle temporal gyrus, right angular gyrus and the cerebellum (Table 3). Across all analyses from independent sites in age-matched samples, the right angular gyrus, left posterior middle temporal gyrus, occipital and temporo-occipital regions were consistently implicated in fluid intelligence subnetwork differences. No subnetworks were identified under the alternate hypothesis in the opposite direction (controls $>$ ASD). In contrast to findings from the younger age cohorts, no subnetworks of increased connectivity associated with fluid intelligence were identified in older individuals with ASD ages 15 to 24 years (site USM; $p>.05$, FWE-corrected). No subnetworks were identified even when the initial

TABLE 2 Nodes identified in atypical subnetwork connectivity association with fluid intelligence in Autism Spectrum Disorders compared to matched controls (Site KKI)

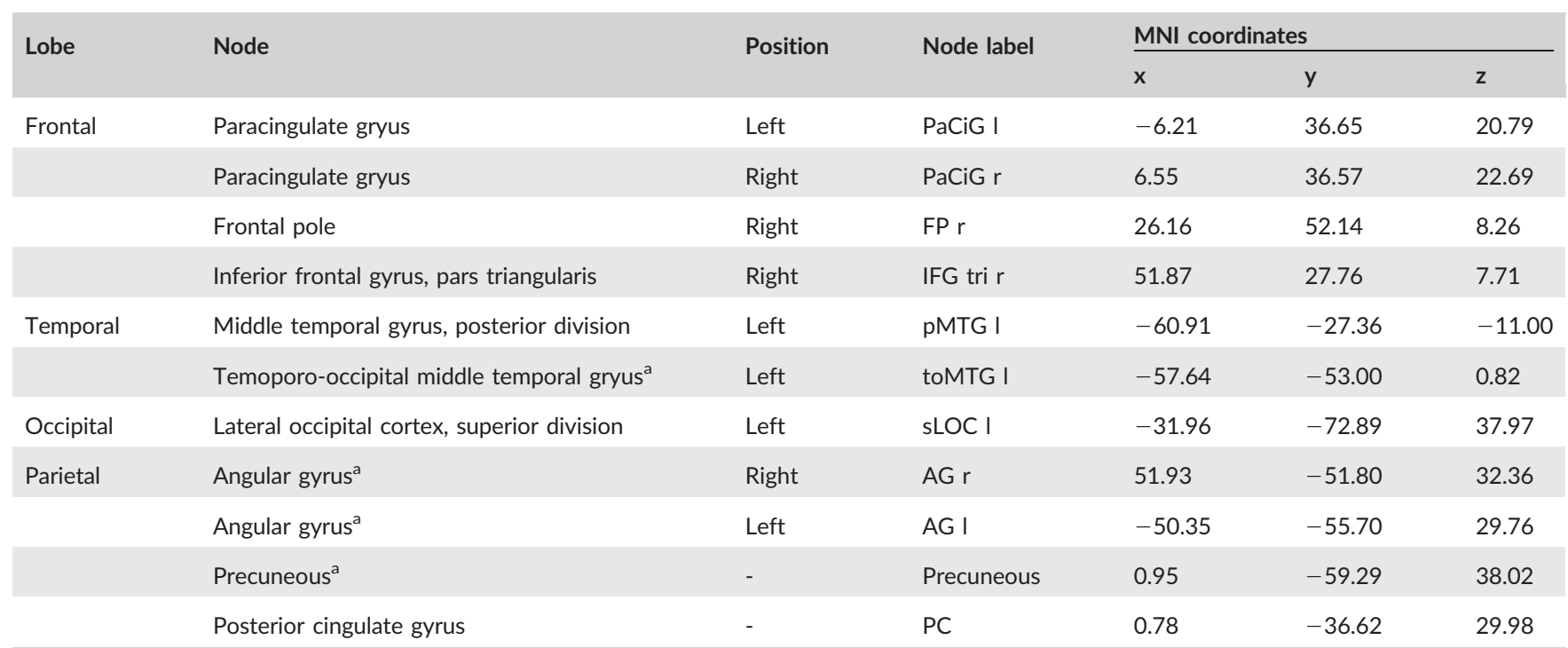

Notes. ${ }^{a}$ Nodes surviving increased thresholding $(T=4)$ in network-based statistics analysis (NBS). $\mathrm{KKI}=$ Kennedy Krieger Institute. 

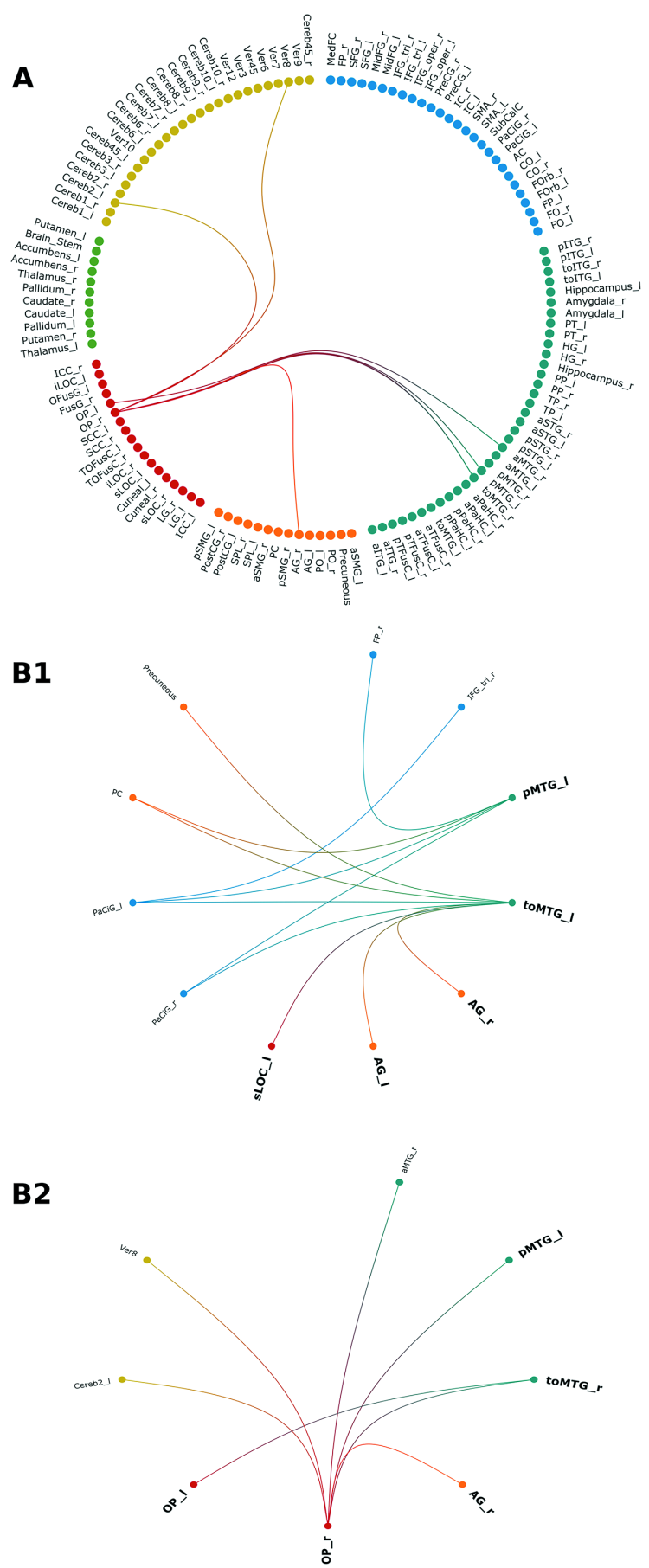

FIGURE 2 (a) Cortical and subcortical regions involved in atypical neural correlates of ASD fluid intelligence in replicated analyses, configured by lobe. The subnetwork represents between-group differences in edge connectivity and fluid intelligence performance association in ASD compared to matched controls (Site: Georgetown University, GU). (b) Network components implicated in atypical neural correlates of ASD fluid intelligence (b1; Site: Kennedy Krieger Institute, $\mathrm{KKI}$ ) and in independent-site replication (b2; Site GU). AG: angular gryus; aMTG: anterior middle temporal gyrus; Cereb2: cerebellum, crus II; FP: frontal pole; IFG: inferior frontal gyrus; OP: occipital pole; PaCiG: paracingulate gyrus; PC: posterior cingulate gyrus; pMTG: posterior middle temporal gyrus; sLOC: Lateral occipital cortex, superior division; toMTG: temporo-occipital middle temporal gyrus; Ver8: cerebellar vermis 8 [Color figure can be viewed at wileyonlinelibrary.com] thresholding of pairwise functional connectivity links and FWEcorrected $p$-values were relaxed.

\section{4 | DISCUSSION}

This is the first study to investigate the neural correlates of fluid intelligence in pediatric ASD cohorts based on a novel brain-behavior matching framework. We identified increased resting state functional connectivity associated with fluid intelligence ability in young ASD males relative to matched controls. These results were replicated in an independent sample with a matched cohort of the same age range. In the context of inter-site differences in sampling, phenotyping, diagnostic classification, MRI scanner and acquisition parameters, and significant heterogeneity within ASD neurobiology, the robustness of present findings suggest the presence of aberrant functional connectivity correlates of fluid intelligence in ASD between 8 to 13 years of age.

Brain regions identified are consistent with previous investigations of localized functional BOLD activity during fluid task performance that show atypical increases in occipito-temporal activity coupled with decreased prefrontal activation in ASD (Yamada, et al., 2012). A recent meta-analysis of grey-matter abnormalities in pediatric ASD reported grey-matter alterations in the right angular gyrus, left inferior occipital gyrus and right inferior temporal gyrus, as well as in frontal, medial parietal and cerebellar regions. Increased grey-matter volume of the right angular gyrus was further associated with increased severity of repetitive behaviors, a core symptom in ASD (Liu, et al., 2017).

In neurotypical individuals, independent component analysis of taskbased fMRI metadata show that temporo-occipital and inferior parietal regions constitute a cluster of intrinsic connectivity networks related to visual perception of complex stimuli higher-level visual processing, visual tracking, mental rotation and spatial discrimination (Laird, et al., 2011). The angular gyrus and temporal-occipital cortex also demonstrate shared activity even among different fluid intelligence tasks, suggesting their role as potential neural correlates of fluid intelligence ability (Ebisch, et al., 2012). In task-free states, connectivity between the angular gyrus and occipital regions form part of a temporally independent functional mode (Smith, et al., 2012). Functionally, the angular gyrus serves as a cross-modal hub that combines and integrates multisensory information for attentional reorientation to critical information, comprehension of environmental events, manipulation of mental representations and problem solving (Seghier, 2013). According to the P-FIT (Parieto-Frontal Integration Theory) hypothesis, the role of the angular gyrus in information processing stages involves integration and abstraction, followed by parietal-frontal interactions that support problem solving and evaluation of solutions (Colom, et al., 2009; Jung \& Haier, 2007). That our findings cohere with known functional connectivity networks of fluid ability in typical controls suggest that these systems also subserve similar functions in ASD, but are more susceptible to aberrations in local and global functional connectivity due to atypical neurodevelopment.

Abnormalities in intrinsic connectivity associated with fluid intelligence were found in younger but not older age groups in the present study. Alterations in brain structure and function in ASD tend to be age- 
TABLE 3 Nodes identified in atypical subnetwork connectivity association with fluid intelligence in Autism Spectrum Disorders compared to matched controls (Site GU)

\begin{tabular}{|c|c|c|c|c|c|c|}
\hline \multirow{2}{*}{ Lobe } & \multirow{2}{*}{ Node } & \multirow{2}{*}{ Position } & \multirow{2}{*}{ Node Label } & \multicolumn{3}{|c|}{ MNI Coordinates } \\
\hline & & & & $\mathrm{x}$ & $\mathrm{Y}$ & z \\
\hline Frontal & - & - & - & - & - & - \\
\hline \multirow{2}{*}{ Temporal } & Middle temporal gyrus, posterior division ${ }^{a}$ & Left & pMTG I & -60.91 & -27.36 & -11.00 \\
\hline & Temporo-occipital middle temporal gyrus ${ }^{a}$ & Right & toMTG $r$ & 58.18 & -49.22 & 1.59 \\
\hline Occipital & Occipital pole & Left & OPI & -16.85 & -96.50 & 6.74 \\
\hline Parietal & Angular gyrus ${ }^{a}$ & Right & $A G r$ & 51.93 & -51.80 & 32.36 \\
\hline
\end{tabular}

Notes. ${ }^{a}$ Nodes consistent across independent-site replication Georgetown University (GU).

dependent, and are characteristic of atypical neurodevelopment in the disorder (Uddin, et al., 2013). In children with ASD, there is significant hypoactivation of the middle frontal gyrus during nonsocial tasks in contrast to adults with ASD (Dickstein, et al., 2013). A similar pattern of early increased functional connectivity followed by a decline in later stages has been reported in other neurodevelopmental disorders, possibly related to dysregulation of brain activity due to aberrant neurodevelopment of structural connectivity of hub regions in the association cortices (Fornito \& Bullmore, 2015). As expected, the inconsistency of abnormalities across different ages presents a significant source of with within- and betweengroup heterogeneity that is difficult to capture reliably.

\section{5 | IMPLICATIONS}

It is of interest that between-group differences in intrinsic connectivity networks associated with fluid intelligence performance were observed between ASD and controls, despite both groups being equivalent on fluid intelligence ability. The degree of association between subnetwork connectivity and fluid ability observed in ASD is therefore more likely related to disorder-specific effects, rather than differences in fluid performance ability (Gray, et al., 2003; Perfetti, et al., 2009). According to the neural efficiency hypothesis, differential cortical activity can be observed among subjects with discrepant neural resources, despite similar task ability. Abnormally increased activation of brain regions during cognitive tasks in atypical populations may indicate a mechanism of neural compensation to achieve the same degree of performance. Consistent with present findings, the process often involves mediation by hub nodes that integrate multiple neural systems, such as the angular gryus in the parietal association cortex. Dedifferentiation, the failure of neural processes to specialize due to neurodevelopmental abnormalities could also underlie early aberrant increases in hub activity observed in our findings (Fornito, et al., 2017). Under this framework of neural compensation, our results also complement previous findings of increased BOLD signal changes with increased fluid task difficulty in the inferior parietal lobule including the angular gyrus, and the left temporo-occipital junction in healthy individuals (Preusse, et al., 2011). Increased resting-state connectivity was also associated with higher intelligence scores (Hearne, et al., 2016a). Together, atypically increased strength of association in the ASD fluid intelligence subnetwork may reflect a compensatory effect to achieve the same level of fluid task performance as ability-matched controls in our analyses.

Consequently, the common assumption that matching on group variables is sufficient to control for all associated variation is problematic, and could introduce artefactual differences in case-control comparisons biased by differential associations in neural correlates (Lefebvre, et al., 2015). As we have shown in this study, group differences in brain structure and function should also demonstrate covariation with variables of interest such as clinical symptom severity. Understanding how alterations in the brain relate to individual differences in phenotypic expression in ASD may reveal important pathways in the etiology of ASD (Picci, et al., 2016). These cohort-dependent findings in ASD highlight the importance of ensuring that differences observed are associated with the effect of interest, rather than variation related to confounds (Bölte, et al., 2009; Dawson, et al., 2007). Given that we have investigated higher functioning ASD subgroups, further work should determine if similar patterns of alterations can be observed in low functioning individuals.

Final considerations are the constraints of NBS. The technique yields increased power over link-based FWE control to detect connected components with whole-brain multiple comparisons, but at the cost of localizing resolution for independent links (Zalesky, et al., 2010). We have thus refrained from directly interpreting individual edge links in identified subnetworks. Connectivity strength and network topology are distinct properties of the brain connectome that can demonstrate mutually exclusive perturbations (Hong, et al., 2013). While we have focused on functional connectivity analyses, our findings establish a promising framework for subsequent investigations into the multi-scale 
configuration of the neural correlates of cognition in various disorders, and across different neuroimaging modalities. As our pipeline only included one method of parcellation, replication of analyses with different parcellation techniques could also strengthen present findings by reducing the likelihood of atlas selection biases (Abraham, et al., 2016).

Apart from identifying fundamental units or collective features of network topology, graph-based measures allow the identification of intermediate mesoscale structures through community detection techniques and across multiple timescales. Importantly, the function of network nodes may differ depending on the scale of analysis (Betzel \& Bassett, 2017). Given also that structural connectivity correlates were not investigated in the present study, cross-modal network analyses that integrate structural and functional data will be crucial to delineate the mechanisms of cognitive development, and how the nature and developmental trajectories of the neural correlates of cognition are altered over time in neurodevelopmental conditions (Grayson \& Fair, 2017). Because anatomical networks determine pathways of neuronal signaling, structural network development likely precedes the complete deployment of global intrinsic functional connectivity networks underlying intellectual ability in children and adolescents. (Petersen \& Sporns, 2015; Vertes \& Bullmore, 2015). The characterization of the neural architecture of intelligence in both typical and atypical neurodevelopmental populations requires further exploration of brain structural and functional network connectivity and topology across multiple scales, and the integration of neuroimaging findings with valid measurement of cognitive constructs of interest.

\section{6 | CONCLUSION}

We demonstrate novel preliminary evidence with replication for an atypical intrinsic connectivity subnetwork associated with fluid intelligence in male ASD children compared to controls. For this particular age cohort, the neural architecture of fluid intelligence in ASD children involves aberrant network integration of distributed regions and an increased strength of association to support equal task performance with same-aged peers. There is potential for longitudinal investigations to delineate inter- and intra-individual variation and between-sex differences in the neurodevelopment of cognitive ability across different populations.

\section{ACKNOWLEDGMENTS}

Data analysis and interpretation was conducted within the Developmental Imaging research group, Murdoch Children's Research Institute and the Children's MRI Centre, Royal Children's Hospital, Melbourne, Victoria. All procedures were conducted in accordance with the Declaration of Helsinki, and approved by The Royal Children's Hospital Human Research Ethics Committee. Data used in this research study was acquired from the public-access Autism Brain Imaging Data Exchange (ABIDE; http://fcon_1000.projects.nitrc.org/ indi/abide/) funded by the National Institute of Mental Health (NIMH 5R21MH107045), and available through the link provided.
The research was supported by the Murdoch Children's Research Institute, the Royal Children's Hospital, Department of Paediatrics The University of Melbourne and the Victorian Government's Operational Infrastructure Support Program. The project was generously supported by $\mathrm{RCH} 1000$, a unique arm of The Royal Children's Hospital Foundation devoted to raising funds for research at The Royal Children's Hospital. The authors declare no potential conflicts of interest with respect to the research, authorship, and/or publication of this article.

\section{ORCID}

Emmanuel Peng Kiat Pua (D) http://orcid.org/0000-0001-9519-2495

\section{REFERENCES}

Aarabi, M. H., Kamalian, A., Mohajer, B., Shandiz, M. S., Eqlimi, E., Shojaei, A., \& Safabakhsh, H. (2015, August) A statistical approach in human brain connectome of Parkinson Disease in elderly people using Network Based Statistics. In Engineering in Medicine and Biology Society (EMBC), 2015 37th Annual International Conference of the IEEE (p 4310-4313). IEEE.

Abraham, A., Milham, M., Martino, A. D., Craddock, R. C., Samaras, D., Thirion, B., \& Varoquaux, G. (2016). Deriving reproducible biomarkers from multi-site resting-state data: An autism-based example. Neurolmage, 147, 736-745. https://doi.org/10.1016/j.neuroimage.2016.10.045

Ahuja, R. K., Magnanti, T. L., \& Orlin, J. B. (1993). Network flows: Theory, algorithms, and applications.

Basten, U., Hilger, K., \& Fiebach, C. J. (2015). Where smart brains are different: A quantitative meta-analysis of functional and structural brain imaging studies on intelligence. Intelligence, 51, 10-27.

Behzadi, Y., Restom, K., Liau, J., \& Liu, T. T. (2007). A component based noise correction method (CompCor) for BOLD and perfusion based fMRI. Neurolmage, 37(1), 90-101.

Betzel, R. F., \& Bassett, D. S. (2017). Multi-scale brain networks. NeuroImage. 160, 73-83.

Bölte, S., Dziobek, I., \& Poustka, F. (2009). Brief report: The level and nature of autistic intelligence revisited. Journal of Autism and Developmental Disorders, 39(4), 678-682.

Burgaleta, M., MacDonald, P. A., Martínez, K., Roman, F. J., Álvarez-Linera, J., González, A. R., ... Colom, R. (2014). Subcortical regional morphology correlates with fluid and spatial intelligence. Human Brain Mapping, 35(5), 1957-1968.

Colom, R., Haier, R. J., Head, K., Álvarez-Linera, J., Quiroga, M. Á., Shih, P. C., \& Jung, R. E. (2009). Gray matter correlates of fluid, crystallized, and spatial intelligence: Testing the P-FIT model. Intelligence, 37 (2), 124-135.

Dawson, M., Soulieres, I., Gernsbacher, M. A., \& Mottron, L. (2007). The level and nature of autistic intelligence. Psychological Science, 18(8), 657-662.

Di Martino, A., Yan, C. G., Li, Q., Denio, E., Castellanos, F. X., Alaerts, K., ... Milham, M. P. (2014). The autism brain imaging data exchange: Towards a large-scale evaluation of the intrinsic brain architecture in autism. Molecular Psychiatry, 19(6), 659-667.

Dickstein, D. P., Pescosolido, M. F., Reidy, B. L., Galvan, T., Kim, K. L., Seymour, K. E., ... Barrett, R. P. (2013). Developmental meta-analysis of the functional neural correlates of autism spectrum disorders. Journal of the American Academy of Child and Adolescent Psychiatry, 52(3), 279-289 e16. 
Ebisch, S. J., Perrucci, M. G., Mercuri, P., Romanelli, R., Mantini, D., Romani, G. L., ... Saggino, A. (2012). Common and unique neuro-functional basis of induction, visualization, and spatial relationships as cognitive components of fluid intelligence. Neurolmage, 62(1), 331-342.

Ehlers, S., Nydén, A., Gillberg, C., Sandberg, A. D., Dahlgren, S. O., Hjelmquist, E., \& Odén, A. (1997). Asperger syndrome, autism and attention disorders: A comparative study of the cognitive profiles of 120 children. Journal of Child Psychology and Psychiatry, 38(2), 207-217.

Fornito, A., \& Bullmore, E. T. (2015). Reconciling abnormalities of brain network structure and function in schizophrenia. Current Opinion in Neurobiology, 30, 44-50.

Fornito, A., Bullmore, E. T., \& Zalesky, A. (2017). Opportunities and Challenges for Psychiatry in the Connectomic Era. Biological Psychiatry: Cognitive Neuroscience and Neuroimaging, 2(1), 9-19.

Fornito, A., Zalesky, A., \& Bullmore, E. (2016). Fundamentals of brain network analysis. San Diego, United States: Academic Press.

Geake, J. G., \& Hansen, P. C. (2010). Functional neural correlates of fluid and crystallized analogizing. Neurolmage, 49(4), 3489-3497.

Gong, Q.-Y., Sluming, V., Mayes, A., Keller, S., Barrick, T., Cezayirli, E., \& Roberts, N. (2005). Voxel-based morphometry and stereology provide convergent evidence of the importance of medial prefrontal cortex for fluid intelligence in healthy adults. Neurolmage, 25(4), 1175-1186.

Gray, J. R., Chabris, C. F., \& Braver, T. S. (2003). Neural mechanisms of general fluid intelligence. Nature Neuroscience, 6(3), 316-322.

Grayson, D. S., \& Fair, D. A. (2017). Development of large-scale functional networks from birth to adulthood: A guide to neuroimaging literature. Neurolmage, 160, 15-31.

Haász, J., Westlye, E. T., Fjær, S., Espeseth, T., Lundervold, A., \& Lundervold, A. J. (2013). General fluid-type intelligence is related to indices of white matter structure in middle-aged and old adults. Neurolmage, 83, 372-383.

Happé, F. G. (1994). Wechsler IQ profile and theory of mind in autism: A research note. Journal of Child Psychology and Psychiatry, 35(8), 1461-1471.

Hayashi, M., Kato, M., Igarashi, K., \& Kashima, H. (2008). Superior fluid intelligence in children with Asperger's disorder. Brain and Cognition 66(3), 306-310.

Hearne, L. J., Mattingley, J. B., \& Cocchi, L. (2016a). Functional brain networks related to individual differences in human intelligence at rest. Sci Rep, 6, 32328.

Hearne, L. J., Mattingley, J. B., \& Cocchi, L. (2016b). Functional brain networks related to individual differences in human intelligence at rest. Scientific Reports, 6, 32328.

Ho, D., Imai, K., King, G., \& Stuart, E. (2011). Matchlt: Nonparametric preprocessing for parametric causal inference. Journal of Statistical Software, 42(8), 1-28.

Hong, S.-B., Zalesky, A., Cocchi, L., Fornito, A., Choi, E.-J., Kim, H.-H., ... Yi, S.-H. (2013). Decreased functional brain connectivity in adolescents with internet addiction. PLOS ONE, 8(2), e57831.

Jung, R. E., \& Haier, R. J. (2007). The Parieto-Frontal Integration Theory (P-FIT) of intelligence: Converging neuroimaging evidence. Behavioral and Brain Sciences, 30(02), 135-154.

Korgaonkar, M. S., Fornito, A., Williams, L. M., \& Grieve, S. M. (2014). Abnormal structural networks characterize major depressive disorder: A connectome analysis. Biological Psychiatry, 76(7), 567-574.

Koshino, H., Carpenter, P. A., Minshew, N. J., Cherkassky, V. L., Keller, T. A., \& Just, M. A. (2005). Functional connectivity in an fMRI working memory task in high-functioning autism. Neurolmage, 24(3), 810-821.
Kosslyn, S. M., Cacioppo, J. T., Davidson, R. J., Hugdahl, K., Lovallo, W. R., Spiegel, D., \& Rose, R. (2002). Bridging psychology and biology: The analysis of individuals in groups. American Psychologist, 57(5), 341.

Kroger, J. K., Sabb, F. W., Fales, C. L., Bookheimer, S. Y., Cohen, M. S., \& Holyoak, K. J. (2002). Recruitment of anterior dorsolateral prefrontal cortex in human reasoning: A parametric study of relational complexity. Cerebral Cortex, 12(5), 477-485.

Laird, A. R., Fox, P. M., Eickhoff, S. B., Turner, J. A., Ray, K. L., McKay, D. R., ... Fox, P. T. (2011). Behavioral interpretations of intrinsic connectivity networks. Journal of Cognitive Neuroscience, 23(12), 40224037.

Langeslag, S. J., Schmidt, M., Ghassabian, A., Jaddoe, V. W., Hofman, A., van der Lugt, A., ... White, T. J. (2013). Functional connectivity between parietal and frontal brain regions and intelligence in young children: The Generation R study. Human Brain Mapping, 34(12), 3299-3307.

Lefebvre, A., Beggiato, A., Bourgeron, T., \& Toro, R. (2015). Neuroanatomical diversity of corpus callosum and brain volume in autism: Meta-analysis, analysis of the autism brain imaging data exchange project, and simulation. Biological Psychiatry, 78(2), 126-134.

Liu, J., Yao, L., Zhang, W., Xiao, Y., Liu, L., Gao, X., ... Gong, Q. (2017). Gray matter abnormalities in pediatric autism spectrum disorder: $\mathrm{A}$ meta-analysis with signed differential mapping. Eur Child Adolesc Psychiatry, 26(8), 1-13.

Lowe, M. J., Sakaie, K. E., Beall, E. B., Calhoun, V. D., Bridwell, D. A., Rubinov, M., \& Rao, S. M. (2016). Modern methods for interrogating the human connectome. Journal of the International Neuropsychological Society, 22(2), 105-119.

Malpas, C. B., Genc, S., Saling, M. M., Velakoulis, D., Desmond, P. M., \& O'Brien, T. J. (2016). MRI correlates of general intelligence in neurotypical adults. Journal of Clinical Neuroscience: Official Journal of the Neurosurgical Society of Australasia, 24, 128-134.

Mottron, L., Bouvet, L., Bonnel, A., Samson, F., Burack, J. A., Dawson, M., $\&$ Heaton, P. (2013). Veridical mapping in the development of exceptional autistic abilities. Neuroscience \& Biobehavioral Reviews, 37, 209-228.

Penke, L., Maniega, S. M., Bastin, M., Hernandez, M. V., Murray, C., Royle, N., ... Deary, I. (2012). Brain white matter tract integrity as a neural foundation for general intelligence. Molecular Psychiatry, 17 (10), 1026-1030.

Perfetti, B., Saggino, A., Ferretti, A., Caulo, M., Romani, G. L., \& Onofrj, M. (2009). Differential patterns of cortical activation as a function of fluid reasoning complexity. Human Brain Mapping, 30(2), 497-510.

Petersen, S. E., \& Sporns, O. (2015). Brain networks and cognitive architectures. Neuron, 88(1), 207-219.

Picci, G., Gotts, S. J., \& Scherf, K. S. (2016). A theoretical rut: Revisiting and critically evaluating the generalized under/over-connectivity hypothesis of autism. Developmental Science, 19(4), 524-549.

Prabhakaran, V., Smith, J. A., Desmond, J. E., Glover, G. H., \& Gabrieli, J. D. (1997). Neural substrates of fluid reasoning: An fMRI study of neocortical activation during performance of the Raven's Progressive Matrices Test. Cognitive Psychology, 33(1), 43-63.

Preusse, F., van der Meer, E., Deshpande, G., Krueger, F., \& Wartenburger, I. (2011). Fluid intelligence allows flexible recruitment of the parieto-frontal network in analogical reasoning. Frontiers in Human Neuroscience, 5, 22.

Pua, E. P. K., Bowden, S. C., \& Seal, M. L. (2017). Autism spectrum disorders: Neuroimaging findings from systematic reviews. Research in Autism Spectrum Disorders, 34, 28-33. 
Reynolds, M. R., \& Keith, T. Z. (2017). Multi-group and hierarchical confirmatory factor analysis of the Wechsler Intelligence Scale for Children-Fifth Edition: What does it measure? Intelligence, 62, 31-47.

Sahyoun, C. P., Belliveau, J. W., Soulières, I., Schwartz, S., \& Mody, M. (2010). Neuroimaging of the functional and structural networks underlying visuospatial vs. linguistic reasoning in high-functioning autism. Neuropsychologia, 48(1), 86-95.

Schneider, W., \& McGrew, K. (2012). The Cattell-Horn-Carroll model of intelligence. In D. P. Flanagan, \& P. L. Harrison (Eds.), Contemporary intellectual assessment: Theories, tests, and issues (pp. 99-144). New York, NY: Guilford press.

Seghier, M. L. (2013). The angular gyrus: Multiple functions and multiple subdivisions. Neuroscientist, 19(1), 43-61.

Simard, I., Luck, D., Mottron, L., Zeffiro, T. A., \& Soulières, I. (2015). Autistic fluid intelligence: Increased reliance on visual functional connectivity with diminished modulation of coupling by task difficulty. Neurolmage: Clinical, 9, 467-478.

Smith, S. M., Fox, P. T., Miller, K. L., Glahn, D. C., Fox, P. M., Mackay, C. E., ... Laird, A. R. (2009). Correspondence of the brain's functional architecture during activation and rest. Proceedings of the National Academy of Sciences, 106(31), 13040-13045.

Smith, S. M., Miller, K. L., Moeller, S., Xu, J., Auerbach, E. J., Woolrich, M. W., ... Glasser, M. F. (2012). Temporally-independent functional modes of spontaneous brain activity. Proceedings of the National Academy of Sciences, 109(8), 3131-3136.

Soulières, I., Dawson, M., Samson, F., Barbeau, E. B., Sahyoun, C. P., Strangman, G. E., ... Mottron, L. (2009). Enhanced visual processing contributes to matrix reasoning in autism. Human Brain Mapping, 30 (12), 4082-4107.

Tavor, I., Jones, O. P., Mars, R., Smith, S., Behrens, T., \& Jbabdi, S. (2016). Task-free MRI predicts individual differences in brain activity during task performance. Science, 352(6282), 216-220.

Team, R. C. (2014). R: A language and environment for statistical computing. R Foundation for Statistical Computing, Vienna, Austria. 2013.

Tzourio-Mazoyer, N., Landeau, B., Papathanassiou, D., Crivello, F., Etard, O., Delcroix, N., ... Joliot, M. (2002). Automated anatomical labeling of activations in SPM using a macroscopic anatomical parcellation of the MNI MRI single-subject brain. Neurolmage, 15(1), 273-289.

Uddin, L. Q., Supekar, K., \& Menon, V. (2013). Reconceptualizing func tional brain connectivity in autism from a developmental perspective. Frontiers in Human Neuroscience, 7 ,

Vertes, P. E., \& Bullmore, E. T. (2015). Annual research review: Growth connectomics-the organization and reorganization of brain networks during normal and abnormal development. Journal of Child Psychology and Psychiatry, 56(3), 299-320.

Whitfield-Gabrieli, S., \& Nieto-Castanon, A. (2012). Conn: A functional connectivity toolbox for correlated and anticorrelated brain networks. Brain Connectivity, 2(3), 125-141.

Yamada, T., Ohta, H., Watanabe, H., Kanai, C., Tani, M., Ohno, T., ... Hashimoto, R. (2012). Functional alterations in neural substrates of geometric reasoning in adults with high-functioning autism. PloS One, 7(8), e43220.

Zalesky, A., Fornito, A., \& Bullmore, E. T. (2010). Network-based statistic: Identifying differences in brain networks. Neurolmage, 53(4), 11971207.

Zalesky, A., Fornito, A., Seal, M. L., Cocchi, L., Westin, C.-F., Bullmore, E. T., ... Pantelis, C. (2011). Disrupted axonal fiber connectivity in schizophrenia. Biological Psychiatry, 69(1), 80-89.

\section{SUPPORTING INFORMATION}

Additional Supporting Information may be found online in the supporting information tab for this article.

How to cite this article: Pua EPK, Malpas CB, Bowden SC, Seal ML. Different brain networks underlying intelligence in autism spectrum disorders. Hum Brain Mapp. 2018;39:3253-3262. https://doi.org/10.1002/hbm.24074 